\title{
Enfermedad periodontal como factor de riesgo para presentar resultados perinatales adversos
}

\author{
José de Jesús Zermeño N. ${ }^{1}$, Clara del Carmen Flores A. ${ }^{1}$, Donato Saldívar $R^{1}{ }^{1}$, \\ Juan Antonio Soria L. 1, Miriam Garza R. 2a, José Luis Iglesias B. 1 \\ 1 Departamento de Ginecología y Obstetricia, Hospital Universitario “Dr. José Eleuterio González”, Universidad Autóno- \\ ma de Nuevo León. Monterrey, Nuevo León, México. \\ 2 Servicio de Biología Molecular, Facultad de Odontología, Universidad Autónoma de Nuevo León. Monterrey, Nuevo \\ León, México.
}

a Odontóloga

\section{RESUMEN}

Objetivo: Determinar si la enfermedad periodontal (EP) es un factor de riesgo que pudiera ejercer alguna influencia en la presentación de resultados perinatales adversos en la población general. Método: Estudio prospectivo de cohorte, en el cual se incluyeron 282 pacientes que acudieron al Hospital Universitario "Dr. José Eleuterio González", Monterrey, Nuevo León, México, para atención del parto. Se realizó examen dental clasificándolas como sanas o enfermas. Para el análisis estadístico se utilizó estadística descriptiva, medidas de tendencia central y de dispersión, $x^{2}$ y riesgo relativo (RR) para establecer asociación entre la enfermedad periodontal y las variables de estudio. Resultados: La incidencia de EP fue de $15 \%$. La edad promedio de 22,1 años. El RR para parto prematuro fue 0,67 (IC 95\% 0,19-2,33; $p=0,81$ ), preeclampsia 1,17 (IC 95\% 0,45-3,02; $p=0,35$ ), ruptura prematura de membranas 0,842 (IC 95\% 0,80-0,88; $p=0,76$ ), bajo peso al nacer 0,85 (IC 95\% 0,33-2,51; p=0,71). Conclusión: En nuestro estudio no encontramos asociación entre pacientes con enfermedad periodontal y resultados perinatales adversos.

\section{PALABRAS CLAVE: Enfermedad periodontal, embarazo, resultados perinatales}

\section{SUMMARY}

Objective: To determine the relationship between maternal periodontal disease and the development of adverse perinatal outcomes. Methods: A prospective cohort study was performed; 282 patients were included, who were assisted for their birth delivery at the Hospital Universitario "Dr. José Eleuterio González", Monterrey, Nuevo León, Mexico. A dental examination was done classifying them as healthy or sick. A descriptive and statistic study was done looking for the association between periodontal disease and the variables of the study were compared using $x^{2}$ and relative risk. Results: The incidence of periondontal disease was $15 \%$. The average age was 22.1 years. The risk ratio for preterm delivery was $0.67(\mathrm{Cl} 95 \% 0.19-2.33 ; \mathrm{p}=0.81)$, preeclampisa $1.17(\mathrm{Cl} 95 \% 0.45-3.02 ; p=0.35)$, premature rupture of membranes $0.842(\mathrm{Cl} 95 \% 0.80-0.88$; $\mathrm{p}=0.76$ ), low birthweight 0.85 ( $\mathrm{Cl} 95 \% 0.33-2.51 ; \mathrm{p}=0.71)$. Conclusion: No significant association was seen between periodontal disease and the adverse perinatal outcomes.

KEY WORDS: Periodontal disease, pregnancy, perinatal outcomes 


\section{INTRODUCCIÓN}

La enfermedad periodontal es una de las enfermedades crónicas de origen infeccioso más comunes en humanos, con una prevalencia que varía entre $10-60 \%$ de acuerdo a los criterios utilizados para definirla. Se puede clasificar como gingivitis (inflamación de los tejidos blandos que rodean al diente) o periodontitis (que involucra la destrucción del tejido que rodea al diente) $(1,2,3)$. El origen de la enfermedad periodontal es un crecimiento acelerado de algunas especies bacterianas en su mayoría Gram negativas y anaerobios, provocando la respuesta inflamatoria produciendo una cantidad significativa de sustancias proinflamatorias, principalmente $\mathrm{IL}-1 \mathrm{~B}$, IL-6, prostaglandina E2 y TNF- $\alpha$, que pueden tener efecto sistémico en el huésped, con la subsecuente destrucción del tejido de soporte dental, lo que lleva a las manifestaciones clínicas $(2,4)$.

En los últimos 5 años se ha investigado intensamente sobre la enfermedad periodontal y su asociación con enfermedades sistémicas como la ateroesclerosis, el infarto agudo al miocardio, eventos vasculares cerebrales, diabetes mellitus y su probable asociación con resultados perinatales adversos (5,6). En 1931, Galloway fue el primero en sugerir que la infección periodontal podría jugar un papel en complicaciones del embarazo como aborto, mastitis, flebitis y toxemia del embarazo (preeclampsia) $(2,7)$.

Algunos de los resultados perinatales adversos con los que se ha asociado la enfermedad periodontal son: parto prematuro (PP), peso bajo al nacer (PBN), ruptura prematura de membranas (RPM) y preeclampsia $(1,7,8)$. Todos ellos de gran importancia por las consecuencias, ya que son las patologías obstétricas más frecuentes y conllevan una elevada morbimortalidad materna y fetal $(9,10)$.

En la actualidad existe un creciente interés en la enfermedad periodontal como posible factor de riesgo independiente para presentar resultados perinatales adversos (parto prematuro, bajo peso al nacer, ruptura prematura de membranas y preeclampsia) y cada vez hay más información que apoya la relación de la enfermedad periodontal como un factor de riesgo independiente para la presentación de estas complicaciones, así como información que no ha logrado evidenciar dicha asociación $(5,11,12,13)$.

Debido a la diferencia de opiniones que existe en cuanto a la participación de la enfermedad periodontal en el desarrollo de enfermedades perinatales adversas, se planteó este estudio para conocer si esta enfermedad es un factor de riesgo para las enfermedades antes descritas en nuestra población (14-17).

\section{MATERIAL Y MÉTODO}

Este estudio prospectivo de cohorte fue realizado en el Departamento de Ginecología y Obstetricia del Hospital Universitario "José E. González" de la ciudad de Monterrey, Nuevo León, México, en colaboración con el Servicio de Odontología, en el período comprendido entre agosto de 2005 y marzo de 2006. Se incluyeron todas las pacientes que acudieron a atención de parto ya fuera por vía vaginal o abdominal a nuestra Institución. Se les realizó una historia clínica completa, valorando antecedentes de importancia, evolución de embarazos previos, evolución de embarazo actual y motivo de consulta. Se incluyeron solo aquellas pacientes que presentaran gestación única ya sea de término o pretérmino, excluyendo todas aquellas que presentaran embarazos múltiples, infección materna activa, diabetes, enfermedad materna crónica y tumoraciones pélvicas, tumoración uterina o incompetencia ístmico-cervical, también se excluyeron pacientes que presentaron productos con malformaciones o polihidramnios.

Todas aquellas pacientes con expediente incompleto fueron eliminadas del estudio. Se llenó hoja de recolección de datos basados en la historia clínica gineco-obstétrica realizada por residentes del Departamento de Ginecología y Obstetricia. A las pacientes seleccionadas se les realizó un examen dental evaluando el grado de inflamación gingival, número de dientes presentes, con caries y restaurados.

El diagnóstico de enfermedad periodontal se estableció al encontrar cuatro o más dientes con profundidad de sondeo mayor o igual a $4 \mathrm{~mm}$ y pérdida de la inserción mayor o igual a $3 \mathrm{~mm}$ en los mismos sitios y sangrado al sondeo.

Se realizó análisis estadístico descriptivo (media, mediana, moda) para las variables sociodemográficas. Los resultados se expresan como promedios \pm desviación estándar $( \pm D E)$. Con la finalidad de observar la relación de las variables de estudio con la enfermedad periodontal se realizó la prueba de $x^{2}$ (menarca, ritmo y duración, edad de inicio de vida sexual, número de compañeros sexuales, método de planificación familiar, fecha de última menstruación, fecha probable de parto, control prenatal y número de consultas, así como la evolución de los embarazos previos (parto pretérmino, ruptura prematura de membranas, preeclampsia, bajo peso al nacer) y embarazo actual (infecciones, amenaza de aborto, internamientos). Se calculo el riesgo relativo para los diferentes resultados adversos analizados y se comparo con la información obtenida de la literatura. 


\section{RESULTADOS}

Se estudiaron un total de 282 pacientes de las cuales $48(15,25 \%)$ se diagnosticaron con enfermedad periodontal y $234(84,75 \%)$, se consideraron sanas. El promedio de edad fue $22,15 \pm 5,43$ años siendo la gran mayoría $(65,6 \%)$ pacientes entre los 18 y 35 años, y la media para edad gestacional al momento del estudio 38,2 \pm 2 semanas. Encontramos diferencia estadísticamente significativa entre la edad de las pacientes con enfermedad periodontal $(p<0,001)$ y sin esta, siendo la media de 21,5 años para las pacientes sanas y de 25,4 años para las pacientes enfermas. Otro resultado significativo fue la edad gestacional (sanas: $38,4 \pm 3,1$ semanas y enfermas: $38,6 \pm 2,4$ semanas; $p=0,01$ ). Más de la mitad de la muestra total de pacientes se encontraban en su primera gestación. Se observó que a mayor número de embarazos, mayor fue la prevalencia de enfermedad periodontal $(p<0,001)$. No hubo diferencia estadística significativa para el resto de variables demográficas (Tabla I).

En cuanto a los antecedentes heredo-familiares que se observaron con mayor frecuencia en ambos grupos fueron la diabetes mellitus $(21,76 \%$ y $23,26 \%$, controles y casos, respectivamente $(p=0,08)$ y la hipertensión arterial (10,46\% y 4,65\%, respectivamente $(p=0,06)$. El principal antecedente no patológico fue el tabaquismo, que se presentó en $6,69 \%$ de las pacientes sanas y $4,65 \%$ de los casos con enfermedad periodontal $(p=0,2)$ (Tabla I).

La cantidad de pacientes que refirió contar con control prenatal fue muy similar en ambos grupos (pacientes sanos: $93,72 \%$ y enfermos: $93,02 \%$ ). Al evaluar la calidad del control prenatal se observó que $61,51 \%$ de las pacientes sanas presentaron un control prenatal adecuado, definiendo este como cinco o más citas, mientras que de las pacientes con enfermedad periodontal solo el $55,81 \%$ presentó un control adecuado $(p=0,06)$.

La evaluación del embarazo actual fue normal en la mayoría de las pacientes tanto sanas como con enfermedad periodontal en un $71 \%$ vs $74 \%$ respectivamente (Tabla II). La principal comorbilidad del embarazo fueron las infecciones (vías urinarias) en $20 \%$ para ambos grupos de pacientes $(p=0,8)$.

Al realizar el estudio de asociación entre las complicaciones perinatales y la enfermedad periodontal se observó que el $29,65 \%$ de las pacientes sanas presentaron alguna complicación perinatal, en comparación con el $25,58 \%$ en el grupo de pacientes enfermas $(p=0,34)$. Las complicaciones perinatales en pacientes sanas fueron: parto pretérmino $10 \%$, preeclampsia $12 \%$, y parto prematuro acompañado de RPM 0,8\%; mientras que para las pacientes con enfermedad periodontal fueron: parto pretérmino $6,9 \%$, preeclampsia $13,9 \%$ y parto prematuro acompañado de RPM en el $4,65 \%$ (Figura 1 ).

El porcentaje de partos prematuros fue de $10 \mathrm{y}$ $6,9 \%$ respetivamente; en cuanto al peso promedio del recién nacido fue de $3.146 \pm 649,6 \mathrm{~g}$, observamos que la gran mayoría de ellos presentaron pesos mayores a $2.500 \mathrm{~g}(p=0,45)$, seguido de aquellos que presentaron un peso de 1.500 a $2.500 \mathrm{~g}(p=0,30)$.

Al realizar el cálculo del riesgo relativo de pacientes con enfermedad periodontal y resultados perinatales adversos observamos que el riesgo para parto prematuro fue 0,67 (IC 95\% 0,19-2,33; $p=0,81$ ), bajo peso al nacimiento 0,85 (IC 95\% 0,33-2,51; $p=0,71$ ), preeclampsia 1,17 (IC 95\% 0,45-3,02; $p=0,35$ ), restricción del crecimiento intrauterino 0,84 (IC95\% $0,80-0,88 ; p=0,26)$, ruptura prematura de membranas 0,842 (IC 95\% 0,80-0,88; $p=0,76$ ) y parto prematuro+ruptura prematura de membranas 5,78 (IC95\% 0,79-42,1; p=0,48) (Tabla III).

Tabla I

CARACTERÍSTICAS DEMOGRÁFICAS ENTRE PACIENTES CON Y SIN ENFERMEDAD PERIODONTAL

\begin{tabular}{lccr}
\hline & Sano $(\mathrm{n}=234)$ & Enfermo $(\mathrm{n}=48)$ & Valor $\mathrm{p}$ \\
\hline Edad materna $^{\text {a }}$ & $21,5 \pm 2.1$ & $25,4 \pm 3,4$ & $<0,001$ \\
Gesta $^{\text {a }}$ & $1 \pm 1,8$ & $2 \pm 3,1$ & $<0,001$ \\
Edad gestacional a & $38,4 \pm 3,1$ & $38,6 \pm 2,4$ & 0,01 \\
Escolaridad >2ria $_{\text {Hipertensión crónica }}$ & $54 \%(128)$ & $60 \%(26)$ & 0,17 \\
Diabetes & $10,46 \%(24)$ & $4,65 \%(2)$ & 0,06 \\
Tabaquismo & $21,76 \%(50)$ & $23,26 \%(11)$ & 0,08 \\
Control prenatal adecuado & $6,69 \%(15)$ & $4,65 \%(2,24)$ & 0,2 \\
\hline
\end{tabular}

\footnotetext{
a Mediana
} 
Tabla II

VARIABLES OBSTÉTRICAS Y SU RELACIÓN CON LA ENFERMEDAD PERIODONTAL

\begin{tabular}{llrrrrr}
\hline & & \multicolumn{2}{c}{$\begin{array}{c}\text { Sano } \\
\text { 239 (85\%) }\end{array}$} & \multicolumn{2}{c}{$\begin{array}{c}\text { Enfermo } \\
43(15 \%)\end{array}$} & Valor $p$ \\
\hline Complicaciones del & Normal & 169 & 71 & 32 & 74 & 0,17 \\
embarazo actual & Complicaciones & 70 & 29 & 11 & 26 & 0,34 \\
Edad gestacional & $<28$ & 2 & 1 & 1 & 1 & 0,81 \\
(semanas) & $28-36$ & 41 & 17 & 8 & 19 & 0,23 \\
& $37-41$ & 194 & 81 & 33 & 77 & 0,31 \\
& $\geq 42$ & 2 & 1 & 1 & 1 & 0,12 \\
Peso por grupo & $<1.500$ & 8 & 3 & 2 & 5 & 0,25 \\
(gramos) & $1.500-2.499$ & 21 & 9 & 4 & 9 & 0,30 \\
& $\geq 2.500$ & 210 & 88 & 37 & 86 & 0,45 \\
\hline
\end{tabular}

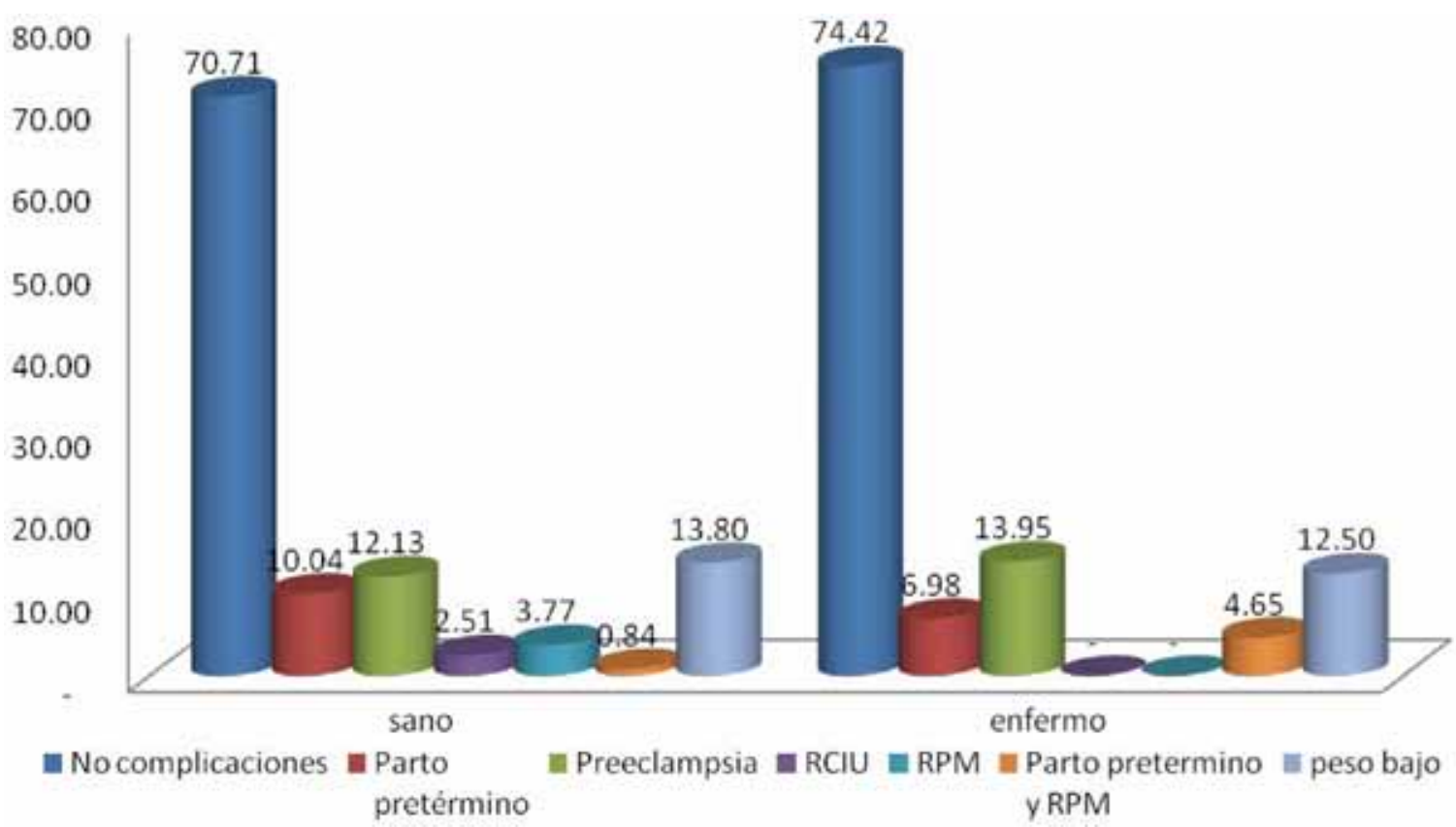

Figura 1. Resultados perinatales adversos en grupo de estudio.

Tabla III

\section{RIESGO RELATIVO (RR) DE LOS DIFERENTES RESULTADOS PERINATALES EN EL GRUPO DE ESTUDIO}

\begin{tabular}{lccc}
\hline Resultado & RR & $95 \%$ IC & Valor $\mathrm{p}$ \\
\hline Parto prematuro & 0,67 & $(0,19-2,33)$ & 0,81 \\
Peso bajo al nacer & 0,85 & $(0,33-2,51)$ & 0,71 \\
Preeclampsia & 1,17 & $(0,45-3,02)$ & 0,35 \\
$\begin{array}{l}\text { Restricción de crecimiento } \\
\text { intrauterino }\end{array}$ & 0,84 & $(0,80-0,88)$ & 0,26 \\
$\begin{array}{l}\text { Ruptura prematura de } \\
\text { membranas (RPM) }\end{array}$ & 0,84 & $(0,80-0,88)$ & 0,76 \\
Parto prematuro y RPM & 5,78 & $(0,79-42,1)$ & 0,48 \\
\hline
\end{tabular}

\section{DISCUSIÓN}

Continua existiendo controversia en la literatura sobre la asociación de la enfermedad periodontal con los diferentes resultados perinatales adversos, mientras en algunos estudios realizados en poblaciones socioeconómicamente deficientes se ha observado la asociación, en otros realizados en países desarrollados como en Europa y Canadá no se ha observado dicha asociación $(16,17,18)$. Como ya se ha mencionado existe evidencia que apunta hacia un proceso infeccioso inflamatorio, como factor clave en el desarrollo de resultados perinatales adversos $(19,20,21)$. La manipulación mecánica puede producir bacteriemia (cepillado), además la 
Tabla IV

ESTUDIOS DE CASOS Y CONTROLES. ANALISIS DE LA RELACIÓN DE ENFERMEDAD PERIODONTAL CON DIFERENTES RESULTADOS PERINATALES ADVERSOS

\begin{tabular}{|c|c|c|c|c|}
\hline $\begin{array}{l}\text { Autor } \\
\text { (referencia) }\end{array}$ & Pacientes & Resultado & $\begin{array}{c}\text { Control } \\
\text { de confusores }\end{array}$ & Conclusión \\
\hline $\begin{array}{l}\text { Offenbacher } \\
2001 \text { (10) }\end{array}$ & $\begin{array}{l}\text { Sanos: } 201 \\
\text { EP leve: } 566 \\
\text { EP severa: } 45\end{array}$ & $\begin{array}{l}\text { PP: } \\
\text { EP leve (OR: } 1,23) \\
\text { EP severa (OR: } 2,12)\end{array}$ & $\mathrm{Si}$ & $\begin{array}{l}\text { Factor de riesgo para PP y } \\
\text { PBN. }\end{array}$ \\
\hline $\begin{array}{l}\text { López } \\
2002 \text { (9) }\end{array}$ & $\begin{array}{l}\text { Grupo } 233 \\
\text { Controles } 406\end{array}$ & $\begin{array}{l}\text { PBPN (RR: 3,5) } \\
\text { PP (RR: } 2,9) \\
\text { PBN (RR: } 3,6)\end{array}$ & $\mathrm{Si}$ & $\begin{array}{l}\text { Posible factor de riesgo para } \\
\text { BPN y PP. }\end{array}$ \\
\hline $\begin{array}{l}\text { Romero } \\
2002(3)\end{array}$ & Casos 69 & $\begin{array}{l}\text { > severidad de en- } \\
\text { fermedad, < peso y } \\
\text { edad gestacional }\end{array}$ & $\mathrm{N} / \mathrm{A}$ & $\begin{array}{l}\text { Riesgo para parto prematuro } \\
\text { y bajo peso al nacer. }\end{array}$ \\
\hline $\begin{array}{l}\text { Boggess } \\
2004(11)\end{array}$ & $\begin{array}{l}496 \text { leve } \\
125 \text { severa } \\
229 \text { controles }\end{array}$ & $\begin{array}{l}\text { Preeclampsia } \\
\text { OR: } 2,4\end{array}$ & $\mathrm{Si}$ & $\begin{array}{l}\text { Riesgo aumentado para } \\
\text { preeclampsia }\end{array}$ \\
\hline $\begin{array}{l}\text { Canakci } \\
2004(12)\end{array}$ & $\begin{array}{l}\text { Casos } 41 \\
\text { Controles } 41\end{array}$ & $\begin{array}{l}\text { Preeclampsia } \\
\text { OR: } 3,47\end{array}$ & $\mathrm{Si}$ & Asociación con preeclampsia \\
\hline
\end{tabular}

enfermedad periodontal crónica puede producir una respuesta inflamatoria sistémica, cuya consecuencia genera niveles elevados de sustancias proinflamatorias $(22,23,24)$.

No existe uniformidad entre los diferentes estudios para definir la enfermedad periodontal, encontrando diferentes índices y cada uno de ellos con diferente sensibilidad y especificidad para definir la enfermedad $(16,22,25)$. Otro posible factor de confusión para el análisis de los diferentes estudios es la falta de control para factores de riesgo ya establecidos (estado socioeconómico bajo, tabaquismo, enfermedad materna crónica, etc.) $(11,14,26)$. De igual manera existe una marcada diferencia para definir efecto perinatal adverso, como son bajo peso al nacer, restricción de crecimiento, parto prematuro, etc. $(27,28)$. Mientras algunos autores han encontrado asociación significativa de la enfermedad periodontal con la presentación de parto prematuro y bajo peso al nacer $(3,7,10)$, mientras que otros, al igual que el estudio realizado en nuestra Institución, no se ha observado dicha asociación $(1,2)$ (Tabla IV). En cuanto a la relación de la enfermedad periodontal con la presentación de preeclampsia, Boggess y cols (11), observaron un incremento del riesgo de 2,4 veces y Canakci y cols (12) de 3,4 veces, en nuestros resultados el incremento del riesgo fue de 1,17 veces, no significativo para el desarrollo de dicha complicación.

\section{CONCLUSIÓN}

En este estudio la enfermedad periodontal no se asoció a un mayor riesgo de resultados perinatales adversos como parto prematuro, RPM pretérmino, preeclampsia y bajo peso al nacimiento.

\section{BIBLIOGRAFÍA}

1. Buduneli N, Baylas H, Buduneli E, Timur T, Dahlen G. Periodontal infection and pre-term low birth weight: a case-control study. J Clin Periodontol 2005;32:174-181.

2. Moore S, Randhawa M, Ide M. A case-control study to investigate an association between adverse pregnancy outcome and periodontal disease. J Clin Periodontol 2005;32:1-5.

3. Romero BC, Chiquito CS, Elejalde LE, Bernardoni CB. Relationship between periodontal disease in pregnant women and the nutritional condition of their newborns. J Periodontol 2002;73:1177-83.

4. Davenport ES, Williams CE, Sterne JA, Murad S, Sivapathasundram B, Curtis MA. Maternal periodontal disease and preterm low birthwight: case-control study. J Dent Res 2002;81:313-8.

5. Xiong X, Buekens P, Fraser WD, Beck J, Offenbacher S. Periodontal disease and adverse pregnancy outcomes: a systematic review. BJOG 2006;113:135-43.

6. Jeffcoat MK. Preterm birth, osteoporosis, and periodontal disease. Compend Contin Educ Dent Suppl 2000;30:5-11.

7. Jeffcoat MK, Geurs NC, Reddy MS, Cliver SP, Gol- 
denberg RL, Hauth JC. Periodontal infection and preterm birth: results of a prospective study. J Am Dent Assoc 2001;132:875-80.

8. Jeffcoat Mk, Geurs Nc, Reddy Ms, Goldenberg RL, Hauth JC. Current evidence regarding periodontal disease as a risk factor in preterm birth. Am Periodontol 2001;6:183-8.

9. López NJ, Smith PC, Gutierrez J. Higher risk of preterm birth and low birth weight in women with periodontal disease. J Dent Res 2002;81:58-63.

10. Offenbacher S, Jared HL, O’Reilly PG, WIls SR, Salvi GE, Lawrence H, Socransky SS, Bech JD. Potential pathogenic mechanisms of periodontitis associated pregnancy complications. Ann Periodont 1998;3:233-50.

11. Boggess KA, Lieff S, Murtha AP, Moss K, Beck J, Offenbacher $\mathrm{S}$. Maternal periodontal disease is associated with an increased risk for preeclampsia. Birth 2004;31:66-7.

12. Canakci V, Canakci CF, Canakci H, Canakci E, Cicek $\mathrm{Y}$, Ingec M, Ozgoz M, Demir T, Dilsiz A, Yagiz $\mathrm{H}$. Periodontal disease as a risk factor for preeclampsia: A case control study. Aust N Z J Obstet Gynaecol 2004;44:568-73.

13. Jarjoura K, Devine P, Perez-Delboy A, Herrera-Abreu M, D'Alton M, Papapanou PN. Markers of periodontal infection and preterm birth. Am J Obstet Gynecol 2005;192:513-9.

14. Goldenberg RL, Culhane JF. Infection as a cause of preterm birth. Clin Perinatol 2003;30:677-700.

15. Ruma M, Boggess K, Moss K, Jared H, Murtha A, Beck J. Maternal periodontal disease, systemic inflammation, and risk for preeclampsia. Am J Obstet Gynecol 2008;198:389.e1-389.e5.

16. Medianos PN, Bobetsis GA, Kinane DF. Is periodontitis associated with an increased risk of coronary heart disease and preterm and/or low birth weight births? J Clin Periodontol 2002;29:22-36.

17. Medianos PN, Lieff S, Murtha AP, Boggess Ka, Auten RI Jr, Beck JD, Offenbacher S. Maternal periodontitis and prematurity. Part II: Maternal infection and fetal exposure. Ann Periodontol 2001;6:175-82.

18. Champagne CM, Medianos PN, Lieff S, Murtha AP, Beck JD, Offenbacher S. Periodontal medicine: emerging concepts in pregnancy outcomes. J Int Acad Periodontol 2000;2:9-13.

19. Dasanayake AP, Boyd D. Medianos PN, Offenbacher $\mathrm{S}$, Hills $\mathrm{E}$. The association between Porphyromonas gingivalis-specific maternal serum IgG and low birth weight. J Periodontol 2001;72:1491-7.

20. Goepgert AR, Jeffcoat MK, Andrews WW, Faye Peterson O, Cliver SP, Goldenberg RL, Hauth JC. Periodontal disease and upper genital tract inflammation in early spontaneous preterm birth. Obstet Gynecol 2004;104:777-83.

21. Teng YT, Taylor GW, Scannapieco F, Kinane DF, Curtis J, Kogon S. Paeriodontal health and systemic disorders. J Can Assoc 2002;68:188-92.

22. Boggess Kim, Beck J, Murtha A, Moss K, Offenbacher $S$. Maternal periodontal disease in early pregnancy and risk for a small for gestational age infant. Am J Obstet Gynecol 2006;194:1316-22.

23. Oettinger-Barak $O$, Barak $S$, Ohel G. Oettinger $M$, Kreutzer H, Peled M, Machtei EE. Severe pregnancy complication (preeclampsia) is associated with greater periodontal destruction. J Periodontol 2005;76:134-7.

24. Shub A, Swain J, Newnham JP. Periodontal disease and adverse pregnancy outcomes. J Matern Fetal Neonatal Med 2006;19:521-8.

25. Clothier B, Stringer M, Jeffcoat MK. Periodontal disease and pregnancy outcomes: exposure, risk and intervention. Best Pract Res Clin Obstet and Gynaecol 2007;20:1-16.

26. Scannapieco FA, Bush RB, Paju S. Periodontal disease as a risk factor for adverse pregnancy outcomes. Ann Periodonto 2003;8:70-8.

27. Vergnes J, Sixou M. Preterm low birth weight and maternal periodontal status: a meta-analysis. Am J Obstet Gynecol 2007;196:135e 1-7.

28. McGaw T. Periodontal disease and preterm delivery of low-birth-wight infants. J Can Assoc 2002;68:165-9. 